Daniela Allocca

Università degli Studi di Napoli "L’Orientale”

\title{
LA RESA DEL MÉTISSAGE RESPIRO, RITMO E RIPETIZIONI: TRADUCENDO KAFKA
}

\section{The surrender of métissage. Breath, rhythm and repetitions: translating Kafka}

\begin{abstract}
In this essay we would like to represent the practice of translation as a process that shows the importance of voice in Kafka's works, voice as sonic body in writing. Beyond the images, theoretical contents, the syntax, the German language reveal the differences the métissage in Kafka's writing. Following Meschonnic's, Spivak's Fortini's translation theories it's possible to outline a translation practice able to restore the differances instead to flatten them.
\end{abstract}

Keywords: Fortini, Kafka, Meschonnic, Spivak, translation, voice

\section{Riassunto}

In questo articolo la pratica della traduzione diventa il processo attraverso il quale rendere evidente l'importanza della voce, del corpo sonoro negli scritti di Kafka.

$\mathrm{Al}$ di là delle immagini e dei contenuti teorici, la sintassi, il tedesco rivelano un lavoro in grado di tracciare le differenze, il métissage della scrittura di Kafka. Seguendo le teorie di Meschonnic, Spivak e Fortini si cerca di delineare una modalità di traduzione che cerchi di ridare la differenza invece di appiattirla.

Parole chiave: Fortini, Kafka, Meschonnic, Spivak, traduzione, voce 
La «grana» è il corpo nella voce che canta, nella mano che scrive, nel membro che esegue.

Barthes, La grana della voce

La scrittura di Kafka secondo Fortini si offre ad una serie infinita di traduzioni. In Gli uomini di Kafka e la critica e le cose, saggio pubblicato all'interno della raccolta Verifica dei poteri Fortini scrive:

La sua opera è infatti l'unica, nel mondo moderno, ad avere dichiaratamente per oggetto il symbolon, la pietruzza segnata da una cifra convenzionale, di cui discorre l'Apocalisse. Avere come soggetto il simbolo, cioè affermare un mondo nel quale ogni cosa e parola, ogni sentimento ed ogni ragione sono segno, sintomo e spia di altro, e dove tutto si trasforma irrimediabilmente, significa davvero scrivere sull'acqua e quindi accettare una infinita glossa, una infinita serie di traduzioni [...] (Fortini, 1989: 264).

Leggere le opere di Franz Kafka significa tradurle in ogni caso, nel senso molteplice che questa pratica comprende ovvero interpretare, riscrivere, trasmutare, aprendosi ai diversi livelli di lettura come sottolinea anche Baioni in Kafka. Letteratura ed ebraismo (Baioni, 1984). In questo caso si è scelto di seguire la traccia delineata dagli elementi attraverso cui è possibile evidenziare il lavoro di Kafka con il respiro, la voce, nel rapporto tra ritmo e ripetizioni si iscrive il rapporto tra la voce come corpo sonoro e la parola come logos (Sparti, 2007: 78-79). Wolf Kittler ha illuminato l'importanza del rapporto tra macchine della scrittura e macchine della fonazione in Kafka ed evidenziato la rilevanza di questi elementi nella sua scrittura in due saggi (Kittler, 1990a: 75-163, 1990b: 383-390), in particolare i racconti del progetto Strafen/Punizioni ruotano intorno a questi elementi. Il progetto, mai edito in tedesco, viene realizzato da Einaudi proprio con le traduzioni di Fortini e comprende i racconti La condanna, La metamorfosi, Nella colonia penale (Fortini, 1997).

In uno scritto dedicato a una analisi delle traduzioni in italiano de $L a$ metamorfosi di Kafka lo stesso Fortini commenta: "Prescelto questo ritmo bisogna sapere che influirà su quel che segue e si trasmetterà ai periodi successivi" (Fortini, 2017: 77). Fortini quindi sottolinea come traduttore la necessità della consapevolezza del ritmo nel testo prodotto dalla traduzione, laddove il lavoro sul ritmo e sulle forme del discorso è centrale nella ricerca di traduttore, in quanto foriero di una indipendenza dal testo 'originale' (Fortini, 2011), sempre tenendo presente la lezione di Benjamin e quindi guardando al parallelismo tra $i$ testi, con le traduzioni che diventano tangenti al testo originale (Benjamin, 1995a: 39-52).

Henri Meschonnic evidenzia quanto il lavoro sul ritmo nella traduzione sia un lavoro che si fa carico della storicità della traduzione, la traduzione secondo 
Meschonnic non ha il compito di "cancellare" le differenze ma di "esporre" le differenze, mostrare l'alterità del testo.

La traduction non comme effacement des différences, mais l'exposition des différences. Non pour transformer les langues. (...) Mais pour situer le métissage, l'altérité infinie des discours, qui sont toujours entre (Meschonnic, 1999: 166).

Le traduzioni in questo ci parlano della relazione tra i testi e il contesto di arrivo e stando alle parole di Fortini, che segue la lezione di Meschonnic, le traduzioni d'autore dovrebbero essere considerate come testo d'autore (Fortini, 2011: 80). La ricerca del corpo sonoro, del ritmo nella scrittura di Kafka diventa ricerca della presenza della voce nella scrittura di Kafka, la traduzione come pratica che si relaziona a questo elemento. Mettersi in relazione con il ritmo dei testi di Kafka in modo da far risuonare le differenze, non addomesticare il ritmo ma relazionarsi al ritmo del testo, far risuonare la grana, la trama che fa di questa letteratura una letteratura minore (Deleuze, Guattari, 1975) capace di dar voce ai plurali dell'identità kafkiana, che si pone come modello delle identità composte e plurime, cifra del contemporaneo.

\section{Voce al limite}

Nel 1910 Kafka aveva iniziato a redigere i Diari. Dopo una conferenza di Rudolf Steiner, fondatore dell'antroposofismo, un movimento che lo affascinò molto, annota:

Im allgemeinen fängt der gesprochene Satz mit seinem großen Anfangsbuchstaben beim Redner an, biegt sich in seinem Verlaufe, so weit er kann, zu den Zuhörern hinaus und kehrt mit dem Schlusspunkt zu dem Redner zurück. Wird aber der Punkt ausgelassen, dann weht der nicht mehr gehaltene Satz unmittelbar mit ganzem Atem den Zuhörer an (Kafka, 1973: 36) ${ }^{1}$.

Ciò che colpisce Kafka, dunque, è il modo in cui il respiro può sostenere l'enunciato, dar vita alle parole fin dove può. L'attenzione viene posta sull'effetto retorico, in che modo modulare il fiato, costruire una frase per far si che essa investa l'uditore. Kafka è molto attento alla dimensione performativa della lettura dei testi.

Egli stesso nel 1912 leggerà in pubblico a Praga La condanna e nel $1919 \mathrm{fu}$ invitato a Monaco a leggere Nella colonia penale, anche in seguito sarà invitato

1 "In genere il periodo parlato incomincia per l'oratore con l'iniziale maiuscola, si piega nello svolgimento fin dove può verso gli ascoltatori e col punto conclusivo ritorna all'oratore. Ma se questo punto viene omesso, il periodo non più trattenuto investe l'uditorio direttamente e d'un fiato"(Kafka, 2002: 147). 
a leggere in pubblico. Egli ama leggere ad alta voce, è un modo per sentirsi parte del testo, e farlo rivivere. Canetti in L'altro processo scrive:

Dice che ancora pochi anni prima si dilettava di sognare di stare leggendo in pubblico l'intera Education sentimentale (il libro di Flaubert che amava con maggior passione) in lingua francese, senza interruzione per tanti giorni e tante notti quante ne sarebbero stati necessari, in una grande sala piena di gente, «da farne riecheggiare le pareti» (Canetti, 1973: 58-59).

Kafka sogna di abbandonarsi completamente alla lettura ad alta voce perché è un modo di immergersi completamente nel teso, e viverlo fisicamente, il suono delle parole dovrebbe avere la forza di investire gli uditori e riempire lo spazio (Cooper, 1991: 19-20). Dato questo interesse di Kafka possiamo dedurre che nella scrittura abbia posto attenzione proprio alla modulazione vocale del testo, all'effetto che l'ascolto del testo potesse avere. Una scrittura costruita in relazione costante con l'uditorio a cui è destinata.

I periodi di ampio respiro, scritti senza punto, tutti d'un fiato, caratteristici della sua scrittura, rivelano il desiderio di investire il lettore con il carico di immagini che essi contengono. Superare i limiti del linguaggio significa anche superare la distanza che la scrittura può creare, per giungere al lettore immediatamente.

I suoi testi implicano che tra essi e la loro "vittima" (l'uditorio) non sussista una distanza stabile, e che essi investano la dimensione affettiva del lettore a un punto tale che questi tema che il raccontato si avventi su di lui, come le locomotive sul pubblico nei recenti film tridimensionali (Adorno, 1972: 262).

L'importanza del suono e della voce viene trattato a fondo anche nel pensiero steineriano. In medicina antroposofica è interessante la nozione di euritmia: si basa sull'uso del suono della voce, della parola per ritrovare un "buon" ritmo, cercando di restaurare l'equilibrio con la natura. L'euritmia curativa è un metodo messo a punto solo nel 1921, dunque in un momento successivo all'incontro e alle riflessioni kafkiane su Steiner, ma il principio dell'euritmia era già presente nella dottrina di Steiner. Anche Kafka è un ricercatore del giusto ritmo per la scrittura, per la sua costruzione. Tullio De Mauro in Minisemantica nota: "Il Produttore non sceglie solo un senso: lo costruisce. Occorre insistere sul carattere per lo meno potenzialmente costruttivo della significazione" (De Mauro, 2001: 182). Questa affermazione è più che mai utile per accedere alla scrittura di Kafka. Da una parte ci sono le immagini che premono per venir incise, dall'altra il lavoro di costruzione, in cui Kafka tende il respiro, cerca di recuperare maggiore spazio possibile come l'animale nel racconto Der Bau/ La costruzione, che lavora per allargare la propria abitazione. "Ich bin auf der Jagd nach der Konstruktionen. Ich komme in ein Zimmer und finde sie in 
einem Winkel weißlich durcheinandergehn" (Kafka, 1973: 207)2 scrive nei Diari. Le costruzioni compaiono ovunque. Kafka le segue e ne viene inseguito. Così costruendo i testi utilizza avverbi, aggettivi, in quantità apparentemente smodata e congiunzioni per conquistare uno spazio che è quello del cammino che compie lo scrittore che insegue la sua visione, ma anche per spostare sempre di più l'arrivo del punto, l'assalto al limite, si compie già a partire dalla sintassi o proprio grazie a questa. Una volta capito il valore di questa costruzione sarà oltremodo importante sottolineare la sua resa nella traduzione laddove spesso si tende a spezzare un pensiero, in questo caso si potrebbe operare in modo tale da restituire pienamente la costruzione iniziale, rispettando la volontà dello scrittore di spostare il punto, allontanare la fine.

Il cammino verso la visione però è anche un cammino tra frammenti, come quello del messaggero in Beim Bau der Chinesischen Mauer /Durante la costruzione della muraglia cinese (Kafka, 1985: 289). Questa lingua mostra le rovine della città, per questo è frammentaria, tagliata, fatta a pezzi, come nota Günther Anders:

Mentre "le frasi" sono uno strumento della comprensione, quindi della vita che continua, rendere attento qualcuno su qualcosa a qualcosa: "Su" e "a" sono delle parti. L'immagine mostra rovine, la frase prende e comunica: "La città distrutta" (Anders, 1951: 54).

La difficoltà insita nel progetto di questa costruzione, l'assalto al limite attraverso uno strumento in rovina come appare il linguaggio in questa descrizione porta lo scrittore in una dimensione dove la scrittura diventa cifra della differenza. Come il respiro così il testo si allarga e si stringe. Lo scrittore forza i limiti del linguaggio realmente costruendo frasi, passaggi nei quali dall'inizio si insegue il senso cercando di superare gli ostacoli, le rovine della città. Se da una parte Kafka vuole incidere il suo corpo nella scrittura attraverso il respiro, la voce, le costruzioni infinite, a perdifiato, d'altra parte è anche la scrittura che è vissuta come qualcosa che può entrare nel corpo, qualcosa di viscerale.

Questa ricerca di corpo, ricordiamo, può essere letta anche nella relazione che Kafka ha con le macchine della scrittura che iniziano a pullulare proprio ad inizio del secolo. La scrittura con la macchina da scrivere è legata allo spazio dell'ufficio mentre quella a mano ad uno spazio intimo. La voce che proviene dalla radio ad esempio è creata dal nostro corpo ma scomposta, trasformata in segnali, ricodificata e riprodotta (Kittler, 1990a: 75-163). La trasmissione non è più affidata alla viva voce del maestro. Questi nuovi strumenti sono strumenti

2 "Vado a caccia di costruzioni. Entro in una stanza e le vedo in un angolo che s'intersecano biancastre” (Kafka, 2002: 391). 
di scrittura della voce. Ma non si tratta né di scrittura né di voce bensì di riproduzione vocale: distanza dalla voce, dal suono, dal corpo.

Il corpo costretto nelle notti alla scrittura è invece carne che in questa scrittura vuole risuonare. Le parole sono sentite come sostanze bio-logiche ed entrano nel corpo così nelle Lettera a Milena: "Danke für das Trotzdem, ein Zauberwort das mir unmittelbar ins Blut eingeht" (Kafka, 2004: 140)3. La parola come una parte viva della propria esistenza, in cui ogni frase può provocare un cambiamento, sempre nella corrispondenza tra Kafka e la sua traduttrice in ungherese, Milena, leggiamo:

Von den Allgemeinheiten die ich bisher über Russland gelesen habe, hat der Beiliegende Aufsatz den größten Eindruck auf mich oder richtiger auf meinen Körper meine Nerven mein Blut gemacht (Kafka, 2004: 238)4

Questa sensibilità per la parola, questo sentire la parola come corpo, come elemento che trapassa il corpo, si rispecchia in una scrittura in cui l'elemento sonoro della parola costruisce il testo come nel racconto Forschungen eines Hundes:

Man umschleicht den Mithund,

man schäumt vor Begierde,

man prügelt sich selbst mit dem eigenen Schwanz,

man fragt,

man bittet,

man heult,

man beißt und erreicht -

und erreicht das, was man

auch ohne jede Anstrengung erreichen würde:

liebevolles Anhören,

freundliche Berührungen,

ehrenvolle Beschnupperungen,

innige Umarmungen,

mein und dein

Heulen mischt sich in eines,

alles ist darauf gerichtet,

ein Entzücken,

Vergessen

und Finden,

3 "Grazie per il Nonostante, parola magica che mi entra direttamente nel sangue" (Kafka, 1988: 116).

4 "Delle cose generiche che ho letto finora sulla Russia, l'articolo incluso mi ha fatto la sua massima impressione o meglio, l'ha fatta al mio corpo, ai miei nervi. Al mio sangue" (Kafka, 1988: 195). 
aber das eine, was man

vor allem erreichen wollte:

Eingeständnis des Wissens, das bleibt versagt

(Kafka, 1985: 333) .

Visualizzando il testo in tal modo si svela una struttura anaforica, che rivela il tessuto poetico di questa prosa. Allitterazioni, assonanze, rime si fanno evidenti. In quanto prosa, invece, la lettura di questo testo diventa una corsa a perdifiato. Lo scrittore si/ci ferma solo quando la vertigine prende il sopravvento e impedisce di andare oltre. La velocità di questa corsa corrisponde alla necessità di inseguimento dei pensieri, per poter afferrare con la voce, quelle fantasie che altrimenti svanirebbero:

Ich erinnere mich an einen Vorfall aus meiner Jugend [...]. Ich glaubte, daß große Dinge um mich vorgehen, deren Anfürer ich sei, denen ich meine Stimme leihen müsse, Dinge, die elend am Boden liegenbleiben müssten, wenn ich nicht für sie lief, für sie meinen Körper schwenkte, nun, Phantasien der Kinder, die mit den Jahren sich verflüchtigen (Kafka, 1985: 325) ${ }^{6}$.

Dar voce alle proprie fantasie, affinché non si disperdano, dar loro il proprio corpo. Il suono delle parole riempie il corpo e così si fa corpo, per questo i cani musici nelle Indagini (...) non "fanno" musica, "sono" musica.

Non parlavano, non cantavano, ma dal nulla facevano prodigiosamente sprigionare la musica. Tutto era musica, il levarsi e il poggiarsi dei loro piedi, determinati movimenti del capo, il loro correre e il loro fermarsi, le posizioni che assumevano l'uno di fronte all'altro, le combinazioni come in una danza, per esempio quando uno poggiava le zampe anteriori sul dorso dell'altro e tutti si mettevano in un ordine per cui il primo che stava in piedi portava il peso di tutti gli altri, oppure quando con $i$ corpi che strisciavano redenti al suolo formavano figure avviluppate

5 "Si striscia intorno al co-cane, si schiuma dal desiderio, si ci flagella con la propria coda, si chiede, si prega, si ulula, si morde e raggiunge - e si raggiunge ciò che si sarebbe potuto raggiungere anche senza alcuno sforzo: ascolto amabile, contatti amichevoli, annusamenti rispettosi, abbracci intimi, il mio e tuo ululato si mescolano divenendo tutt'uno; tutto si dispone a questo scopo, un incanto, dimenticare e trovare, ma ciò che più di tutto si voleva raggiungere, riconoscimento dei saperi, resta bandito.” Traduzione mia.

6 "Ricordo un episodio della mia giovinezza, $[. .$.$] credevo che accadessero grandi$ cose attorno a me, e che io le dirigessi, che dovessi dar loro voce, cose che sarebbero state costrette a rimanere miseramente a terra se non avessi corso per loro, insomma, sarebbero rimaste fantasie di bambini che si disperdono con gli anni" (Kafka, 2000: 19). Grassetto mio nel testo. 
e non sbagliavano mai nemmeno l'ultimo, che era ancora un po' insicuro, che non si trovava sempre a tempo con gli altri, in un certo senso qualche volta esitava quando la melodia attaccava, che però era insicuro solo se confrontato con la grandiosa sicurezza degli altri, e anche se avesse mostrato una insicurezza ben maggiore, ovvero totale, non avrebbe potuto arrecare alcun danno, poiché gli altri, grandi maestri, inesorabili mantenevano il ritmo (Kafka, 2000: 20-21).

Ancora una volta il respiro dello scrittore si tende fino a costruire un periodo al limite del respiro. Come in un una danza sono i movimenti a creare la musica, così nella scrittura le assonanze, le ripetizioni, richiami, allitterazioni, combinazioni delle parole creano una costruzione che può tendere il respiro fino al limite e accompagnare la danza dei suoni sprigionata dal corpo dei cani. Nel testo in tedesco il ritmo è der Takt (Kafka, 1985: 326) che indica «la battuta», «la cadenza $\gg$, il corpo della scrittura e la scrittura del corpo diventano tutt'uno, così come il corpo orale, la voce, il respiro, il ritmo è intessuto in questa scrittura.

\section{Sincretismi linguistici}

Questo racconto secondo Hartmut Binder descriverebbe i tentativi di Kafka nello studio dell'ebraico moderno, così il suo incontro con i cani musici nell'infanzia rappresenterebbe il suo primo incontro con la lingua ebraica. Quando alla fine del racconto parla di un secondo incontro musicale, esso dovrebbe simboleggiare la ripresa di uno studio assiduo dell'ebraico da parte di Kafka. Sin dal 1917 aveva iniziato a studiare l'ebraico da autodidatta grazie al testo di Moses Rath. Il testo era composto di 150 lezioni, Kafka era sicuramente arrivato alla $45^{\mathrm{ma}}$ lezione all'epoca come dimostrano le parole appuntate sui suoi quaderni. Successivamente nel 1923 riprende gli studi dell'ebraico avvalendosi anche delle lezioni di Puah Ben-Tovim, madrelingua ebraica (Binder, 1988: 137-160). Questa interpretazione è sicuramente interessante ma quello che qui si vorrebbe sottolineare è la possibilità che l'incontro con l'ebraico abbia influenzato in altro modo la genesi di questo racconto. I giochi di parole, le permutazioni, le derivazion e le nominalizzazioni che caratterizzano la scrittura di Kafka diventano molto più frequenti proprio nei racconti scritti durante la ripresa dello studio dell'ebraico. La lingua ebraica è una lingua a base radicale. Questo significa che forme verbali, aggettivi e sostantivi vengono formati a partire da una radice, nella maggior parte dei casi in ebraico si tratta di una radice composta da tre consonanti. Lo stesso Binder nota che:

Prendendo spunto dalla trattazione sistematica del verbo nel testo di Rath, si è segnato [Kafka] su dei foglietti lunghi e stretti, che portava sempre con sé e che, perciò, poteva sempre memorizzare, le radici verbali, le loro flessioni, e le derivazioni ulteriori (Binder, 1988: 147). 
Questo gioco tra le radici in ebraico può aver sollecitato il medesimo movimento nella lingua tedesca, da qui l'evidente aumento di catene semantiche create attraverso tutte le possibilità di composizione, derivazione, flessione della lingua tedesca, che si verifica nei suoi ultimi racconti. Come detto abbiamo diverse possibilità di lettura quindi questa musica può essere si quella sprigionata dalla lingua ebraica, come afferma Binder, ma si potrebbe ipotizzare una analogia tra i sette cani, grandi maestri della musica e le sette note musicali tenendo presente il rapporto tra questa scrittura e la tradizione romantica così come delineato nel saggio di Menke Prosopopoiia. Stimme und Text bei Brentano, Hoffmann, Kleist und Kafka (2000). Il piccolo cane riesce a salvarsi, a riprendere fiato, ma alla fine del racconto si imbatte in un altro incontro "musicale". L'incontro con il cacciatore. L'arma di questo cacciatore come quella delle sirene (Kafka, 1985: 304-305) è il non-canto. Prima ancora che l'immagine gli riveli che il cacciatore sta per cantare, prima ancora che iniziasse a sentire qualche accenno di canto, il cane avverte con tutto il corpo che il cacciatore sta per cantare. Sente le vibrazioni della voce. La parola voce in ebraico è ל / qol.

Con qol l'ebraico denomina cioè l'onda emotiva che emana da un'entità e che l'osservatore può cogliere con uno sguardo, ancor prima di percepire un suono. La voce è infatti intesa, nella scrittura, anche come qualità visibile, quasi che il suo echeggiare faccia sorgere, anziché una reazione uditiva, uno stimolo visivo (Busi, 1999: 282).

In ambito ebraico ritroviamo dunque la coscienza della potenza del suono, della sua energia dinamica. L'esperienza del cane con il cacciatore è proprio un presentire la voce, un pre-sentire, il canto del cacciatore come nelle sirene Ulisse pre-vede il canto delle sirene, la scena descrive una sinestesia tra visione e udito, laddove l'immagine del canto produce il canto e lo spazio di enunciazione è sempre anche spazio di audizione. La 'resa' del traduttore è anche un arrendersi al testo, come indicato da Spivak nel suo saggio The politics of translation (Spivak, 1993: 183). Arrendersi alla voce della scrittura di Kafka non significa annullare il testo nella musica, renderlo solo teatro, quanto dar conto della dimensione vibrazionale di questa scrittura:

The contrast between the "organis" of a "vibrant story", in wich the parts fall into place naturally, and the forced "construction" of a text in wich the parts are assembled according to some artificial scheme, plays a central role in his poetics (Cooper, 1991: 40).

Il lavoro sul ritmo in traduzione diventa quindi possibilità per ridare la differenza che la scrittura contiene, restituire il corpo vibrante, il corpo sonoro, il corpo rimosso, facendo in modo che la traduzione sia il risultato di una relazione con il corpo del testo di partenza. Arrendersi ci rende in questo caso consapevoli 
e ci fa produttori di métissage nel modo auspicato da Meschonnic, consapevoli che forse proprio la sensibilità del nostro tempo ci apre a questa dimensione, infatti è nelle arti contemporanee che Kolesch (2006) sottolinea l'influsso dell' «audiovisivo». Non è un caso che quindi la traduzione fortiniana sia quella che ha messo in evidenza il lavoro sul ritmo, ma a sua volta Fortini lavora su Kafka tenendo presente Leopardi (Fichera, 2011), aprendo il lettore italiano a una cifra diversa di questa scrittura. Oggi in un epoca in cui la scrittura plurilinguistica è una pratica diffusa, si può aggiungere alle «infinite traduzioni» di Kafka anche una traduzione che tenga conto delle identità linguistiche sottopelle che diventano parte del tessuto linguistico, del corpo vibrazionale della scrittura di Kafka.

\section{Bibliografia}

Adorno, T.W. (1972): Prismi, Saggi sulla critica della cultura. Torino: Einaudi.

Anders, G. (1951): Kafka pro und contra. München: Verlag C. H. Beck.

Baioni, G. (1984): Kafka: letteratura ed ebraismo. Torino: Einaudi.

Barthes, R. (2001): La grana della voce. In: Barthes, R., L'ovvio e l'ottuso. Saggi critici III, pp. 257-266. Torino: Einaudi.

Benjamin, W. (1995a): Il compito del traduttore. In: Benjamin, W., Angelus Novus, pp. 39-52. Torino: Einaudi.

Benjamin, W. (1995b): Franz Kafka. Per il decimo anniversario della sua morte. In: Benjamin, W., Angelus Novus, pp. 275-305. Torino: Einaudi.

Binder, H. (1988): Studi di ebraico di Kafka. Un tentativo biografico-interpretativo.

In: Cavarocchi, M., La certezza che toglie ogni speranza, Contributi per l'approfondimento dell'aspetto ebraico in Kafka, pp. 137-160. Firenze: La Giuntina. Busi, G. (1999): Simboli del pensiero ebraico. Einaudi: Torino.

Canetti, E. (1973): L'altro processo, Le lettere di Kafka a Felice. Milano: Longanesi.

Cooper, G. von N. (1991): Kafka and Language: In the Stream of Thoughts and Life. California: Adriane Press.

Deleuze, G., Guattari, F. (1975): Kafka. Pour une littérature mineure. Éditions Minuit: Paris.

De Mauro, T. (2001): Minisemantica. Bari-Roma: Laterza.

Fichera, G. (2011): Per una verifica della gioia: Fortini traduce Kafka, http://www. ospiteingrato.unisi.it/per-una-verifica-della-gioia-fortini-traduce-kafka/ (accesso: 28.08.2018).

Fortini, F. (1989): Verifica dei poteri. Torino: Einaudi.

Fortini, F. (1997): Strafen. Punizioni. Torino: Einaudi.

Fortini, F. (2011): Lezioni sulla traduzione. Macerata: Quodlibet.

Fortini, F. (2017): Capoversi su Kafka. Matelica: Hacca. Kafka, F. (1970): Racconti, a cura di E. Pocar. Milano: Mondadori. Kafka, F. (1973): Tagebücher 1910-1923. Frankfurt am Main: Fischer. 
Kafka, F. (1985): Sämtliche Erzählungen, herausgegeben von P. Raabe. Frankfurt am Main: Fischer.

Kafka, F. (1988): Lettere a Milena, a cura di F. Masini. Milano: Mondadori.

Kafka, F. (1995): Racconti, a cura di G. Schiavoni. Milano: Rizzoli.

Kafka, F. (2000): Cinque storie di animali, a cura di C. Miglio. Roma: Donzelli.

Kafka, F. (2002): Diari, a cura di E. Pocar. Mondadori: Milano.

Kafka, F. (2004): Briefe an Milena, Erweiterte Neuausgabe. Frankfurt am Main: Fischer.

Kittler, W. (1990a): Schreibmachinen, Sprechmaschinen. Effekte technischer Medien im Werk Franz Kafkas. In: Kittler, W., Neumann, G., Franz Kafka: Schriftverkehr, pp. 75-163. Freiburg: Rombach Verlag.

Kittler, W. (1990b): His Masters's Voice. Zur Funktion der Musik im Werk Franz Kafkas. In: Kittler, W., Neumann, G., Franz Kafka: Schriftverkehr, pp. 383-390. Freiburg: Rombach Verlag.

Kolesch, D. (2006): Weh sehen will, muss hören. Stimmlichkeit und Visualität in der Gegenwartskunst. In: Kolesch, D., Krämer, S., Stimme, pp. 40-64. Frankfurt am Main: Suhrkamp Verlag.

Menke, B. (2000): Prosopopoiia. Stimme und Text bei Brentano, Hoffmann, Kleist und Kafka. Wilhelm Fink Verlag: München.

Meschonnic, H. (1999): Poétique du traduire. Éditions Verider: Paris.

Sparti, D. (2007): Il corpo sonoro. Oralità e scrittura nel jazz. Bologna: il Mulino.

Spivak, C. (1993): The Politics of Translation. In: Spivak, C., Outside in the Teaching Machine, pp. 179-200. New York: Routledge. 